\title{
Assessing the effect of a single dose florfenicol treatment in feedlot cattle on the antimicrobial resistance patterns in faecal Escherichia coli
}

\author{
Anna Catharina B. BERGE ${ }^{\mathrm{a}}$, William B. EPPERSON ${ }^{\mathrm{b}, \mathrm{c} *}$, \\ Robbi H. PRITCHARD ${ }^{\mathrm{d}}$ \\ a Veterinary Medicine Teaching and Research Center, University of California Davis, 18830 road 112, \\ Tulare, CA, 93274, USA \\ ${ }^{\mathrm{b}}$ Department of Veterinary Science, College of Agriculture and Biological Sciences, South Dakota \\ State University, Brookings, South Dakota, 57007, USA \\ ${ }^{c}$ Present address: Department of Veterinary Preventive Medicine, College of Veterinary Medicine, \\ The Ohio State University, 1920 Coffey road, Columbus, Ohio, USA \\ d Department of Animal and Range Science, College of Agriculture and Biological Sciences, \\ South Dakota State University, North Campus drive, Brookings, South Dakota, 57007, USA
}

(Received 10 August 2004; accepted 21 February 2005)

\begin{abstract}
The objective of this clinical trial was to examine the effect of a single dose of florfenicol on antimicrobial resistance patterns in faecal E. coli of feedlot steers. Steers $(n=370)$, were purchased from two sources and housed in outdoor concrete floored pens. Two cattle from each pen ( $n=42$ pens, 84 cattle) were randomly selected for faecal sampling at study day 1, 14, 28, and 42 . One sampled animal from each of 21 pens was randomly selected to receive a single $39.6 \mathrm{mg} / \mathrm{kg}$ dose of florfenicol subcutaneously at study day 11 . Ten lactose positive colonies were isolated from faecal swabs and tested for antimicrobial resistance to 11 antimicrobials using the disk diffusion method. Zones of inhibition were grouped using cluster analysis and clusters were ordered by increasing multiple resistance. A cumulative logistic regression model using generalized estimating equations was used to assess factors associated with increasing levels of multiple resistance. Immediately post-treatment, all isolates obtained from treated cattle belonged to multiple resistant clusters containing chloramphenicol resistance. Though less pronounced in later sampling, resistance to chloramphenicol and other antimicrobials persisted. Antimicrobial treatment, sampling time and animal source, as well as interactions between these variables, were important predictors of the odds of $E$. coli belonging to a more resistant cluster. A very clear but transitory shift to increasingly multiple resistant faecal $E$. coli in response to florfenicol treatment was observed. There was no indication of horizontal transfer of resistant $E$. coli between steers. Level of resistance was influenced by complex interaction of animal source and previous management.
\end{abstract}

antimicrobial resistance / food animal / florfenicol / Escherichia coli

\section{INTRODUCTION}

There has been ongoing debate concerning the effect of therapeutic and non-therapeutic veterinary antimicrobials on human health [25, 28]. Animal agriculture has been implicated as a major user of antimicrobials, and, by extension, a major contributor to antimicrobial resistance. Traditionally, antimicrobial resistance was studied through

\footnotetext{
* Corresponding author: Epperson.1@ osu.edu
} 
use of a specific organism, and through monitoring antimicrobial resistance in that organism over time [26]. Often these specific organisms were pathogens, and intensive study allowed identification of emerging trends, estimation of prevalence in animals or animal products, and investigation of antimicrobial resistance mechanisms. However, for population assessment, use of a pathogenic organism may be problematic since prevalence may be low [24]. The objective of population monitoring may be more toward determining predominate antimicrobial resistance, rather than documenting low prevalence antimicrobial resistance.

Antimicrobial resistance assessment of commensal organisms has been used to study gastrointestinal tract ecology and track environmental organisms to a presumed source [14, 16, 23]. Commensal $E$. coli have been used as a monitor of antimicrobial resistance in cattle and swine populations [5, 10, 30, 31]. Use of commensal organisms is convenient and allows measurement of the resistance gene pool that may be transmitted to pathogenic bacteria. Several studies have indicated that interspecies resistance transfer occur in bacteria, and increased recovery of antimicrobial resistant faecal $E$. coli was seen in dairy farms affected with resistant salmonellosis $[6,8,11]$. The level of resistance in commensal enteric bacteria has been correlated with resistance in pathogenic bacteria [11].

In cattle, factors apart from antimicrobial administration affect antimicrobial resistance, including age, environment, and nutrition [9, 16-18]. Long-term use of antimicrobials may select for antimicrobial resistance that persists over time, though duration of antimicrobial resistance in faecal E. coli in feedlot cattle following therapeutic antimicrobial usage appears to be short [20, 29].

Florfenicol is a veterinary fluorinated analogue of tiamphenicol approved in 1996 in the United States for bovine respiratory disease pathogens [13]. Florfenicol is a bac- teriostatic antimicrobial with similar mode of action to chloramphenicol, binding to the $50 \mathrm{~S}$ subunit of bacterial ribosomes. The flo gene confers resistance to chloramphenicol and florfenicol, and is most commonly located on a large transferable plasmid in bovine enteric E. coli [27, 33].

The objective of this study was to examine the effect of a single therapeutic dose of florfenicol on antimicrobial susceptibility patterns in faecal $E$. coli of steers within one month post treatment.

\section{MATERIALS AND METHODS}

\subsection{Animal sampling and treatment}

The clinical trial was performed on animals included in nutrition experiments at the South Dakota State University Ruminant Nutrition Research Center in the autumn of 2001. Angus and Angus crossbred steers $(n=370)$, weighing approximately $270 \mathrm{~kg}$ were purchased directly from source A $(n=196)$ and B $(n=174)$ and arrived on October 25 and October 31, 2001 respectively. Calves and dams were rounded up on the range and steer calves were weaned and shipped directly from the ranch. Sixty steers from source B had been weaned and held in a dirt lot for approximately one month prior to shipment to the research center. Cattle were used in two nutrition experiments, apart from the study here. Source A steers were assigned to one of two treatments in a study involving dietary trace mineral inclusion rate. Source B steers were placed into one of four treatments to study effect of various grain byproducts on health and performance. Cattle were housed in 42 open concrete floor pens (8-10 head/pen). Prior to the study, pens had been scraped free of physical debris and were unoccupied for 30 days. The ionophore monensin (Rumensin, Elanco Animal Health, Indianapolis, Indiana, USA), was incorporated into the diet at a rate of 20 grams/ton of feed, and was the only 
Table I. Antimicrobials and concentrations used for susceptibility testing of faecal $E$. coli isolates using the disk diffusion method.

\begin{tabular}{lcc}
\hline \multicolumn{1}{c}{ Antimicrobial } & Code & Concentration $(\mu \mathrm{g})$ \\
\hline Amoxicillin/clavulanic acid & AMC & $20 / 10$ \\
Ampicillin & AMP & 10 \\
Cephalothin & CEF & 30 \\
Chloramphenicol & CHL & 30 \\
Gentamicin & GEN & 10 \\
Nalidixic acid & NAL & 30 \\
Spectinomycin & SPT & 100 \\
Streptomycin & STR & 10 \\
Sulfisoxazole & SULF & 250 \\
Tetracycline & TET & 30 \\
Sulfamethoxazole/trimethoprim & SXT & $23.75 / 1.25$ \\
\hline
\end{tabular}

antimicrobial in feed or water. Two cattle from each pen $(n=84)$ were randomly enrolled into the study. Serial rectal faecal samples were obtained using cotton tipped swabs from all study animals on days 1,14 , 28 , and 42 . At study day 11 , one study animal from half the pens $(n=21)$ was randomly selected to receive a single $39.6 \mathrm{mg} /$ $\mathrm{kg}$ dose of florfenicol (Nuflor - ScheringPlough Animal Health, Kenilworth, New Jersey, USA) by subcutaneous injection. The use of this treatment regime was within label indications. Animals selected for antimicrobial treatment came from both source A $(n=12,25 \%$ of cattle) and source B $(n=9$, $25 \%$ of cattle). A total of 48 steers in 24 pens from source $A$ and 36 steers in 18 pens from source B were sampled.

\subsection{Sampling and isolation of $E$. coli}

Swabs were plated onto MacConkey agar within $3 \mathrm{~h}$ of acquisition and incubated $24 \mathrm{~h}$ at $35^{\circ} \mathrm{C}$. Ten well isolated lactose positive colonies (or as many that were present, if less than 10) were randomly selected from each plate for antimicrobial resistance testing. Biochemical confirmation was performed on 49 randomly selected isolates using triple sugar iron agar (TSI), motility, and indole media.

Antimicrobial susceptibility tests were performed using the disk diffusion assay [4]. The selected colonies were inoculated into $\mathrm{NaCl}$ sterile solution $(0.85 \%)$ to achieve an optical density corresponding to $0.5 \mathrm{McFar}-$ land units. A sterile swab was dipped into the adjusted suspension and streaked onto Mueller-Hinton $(\mathrm{MH})$ agar plates $(150 \times$ $15 \mathrm{~mm}$ ) to form a uniform lawn of bacterial growth. Eleven drug-impregnated disks were placed on the surface of the agar using a disk dispenser (Tab. I). The plates were incubated at $37^{\circ} \mathrm{C}$ for 16 to $18 \mathrm{~h}$ and zones of inhibition around each disc measured to the nearest $\mathrm{mm}$. The quality control strain E. coli ATCC 25922 (ATTC, Manassas, Virginia, USA) was used for each batch of isolates tested [1].

\subsection{Quantitative analysis}

Data from E. coli antibiograms were a series of continuous measurements representing inhibition zone size to each of 11 antimicrobials. Descriptive statistics were calculated on isolates to determine the zone 
Table II. Antimicrobial susceptibility clusters of faecal E. coli isolates (total number $=3145$ ) in a trial evaluating single-dose florfenicol treatment of steers. Mean disk diffusion zone $(\mathrm{mm})$ is given for each cluster-antimicrobial combination, as well as number of isolates (Frequency) in each cluster*.

\begin{tabular}{lcccccccccccc}
\hline Cluster & Frequency & AMC & AMP & CEF & CHL & GEN & NAL & SPT & STR & SXT & SULF & TET \\
\hline A & 2518 & 25 & 23 & 22 & 25 & 23 & 27 & 24 & 18 & 31 & 25 & 26 \\
B & 263 & 25 & 23 & 22 & 25 & 23 & 26 & 24 & 17 & 30 & 25 & 11 \\
C & 23 & 22 & 6 & 21 & 26 & 23 & 27 & 25 & 18 & 31 & 24 & 6 \\
D & 5 & 27 & 26 & 24 & 6 & 22 & 29 & 23 & 6 & 31 & 24 & 6 \\
E & 54 & 24 & 22 & 21 & 25 & 23 & 27 & 24 & 11 & 24 & 6 & 6 \\
F & 31 & 25 & 23 & 22 & 26 & 23 & 28 & 15 & 12 & 26 & 6 & 6 \\
G & 71 & 26 & 24 & 22 & 6 & 23 & 26 & 23 & 6 & 22 & 6 & 7 \\
H & 12 & 23 & 6 & 21 & 26 & 22 & 28 & 15 & 9 & 25 & 6 & 6 \\
I & 12 & 20 & 6 & 20 & 6 & 24 & 26 & 24 & 7 & 21 & 6 & 6 \\
J & 8 & 20 & 6 & 20 & 6 & 22 & 29 & 16 & 6 & 6 & 6 & 6 \\
K & 31 & 11 & 6 & 6 & 6 & 23 & 27 & 25 & 6 & 19 & 6 & 6 \\
L & 107 & 13 & 6 & 6 & 6 & 23 & 28 & 15 & 6 & 23 & 6 & 7 \\
M & 10 & 16 & 6 & 12 & 6 & 12 & 28 & 6 & 6 & 21 & 6 & 6 \\
\hline
\end{tabular}

* Zone sizes in dark shading indicate resistance to the antimicrobial and zone sizes in light shading indicate intermediate resistance, according to National Committee for Clinical Laboratory Standards (NCCLS) guidelines for human E. coli. Zone sizes without shading indicate sensitivity according to same standard.

size distribution for each antimicrobial. Antimicrobials with a bimodal distribution of inhibition zones were used in grouping the strains using cluster analysis. Clusters were determined using Ward's minimum variance method and squared Euclidean distance, as described [5, 12, 32]. Clusters were considered ordinal outcomes, and ordered according to increasing resistance, based on a decreasing sum of mean inhibition zone sizes to the eleven antimicrobials. The statistical software package SAS version 8 (SAS Institute, Cary, North Carolina, USA) was used for data analysis.

Contingency tables of effects of source (A or B), sampling (1, 2, 3 or 4), and florfenicol treatment on $E$. coli antimicrobial cluster membership were developed. The effect of florfenicol treatment on the distribution of chloramphenicol resistance was evaluated with the Chi-square statistic. The Wilcoxon-Mann-Whitney test was used to determine differences in resistance between source groups. A comparison of antimicrobial resistance clusters between steers being housed in the same pen as treated steers and those steers in pens without any treated steers was performed using stratified analysis, and post-antibiotic treatment cluster distribution was tested using the WilcoxonMann-Whitney test.

A mixed effects cumulative logistic regression model using generalized estimating equations was developed, using cluster membership as the outcome of interest $[3,19]$. The model predicted the odds of the $E$. coli belonging to a more resistant cluster, given a set of explanatory variables including source ("source", consisting of two categories [A or B]), early weaning (termed "wean", consisting of two categories to account for steers from source B that were weaned one month prior to shipment and other), sampling time ("time", 1-4), 
florfenicol treatment ("abtx", yes/no categorical), pen mate to treated steer (yes/no categorical), and nutrition treatment (two categories). Second and third order interactions were included in the model. A stepwise forward selection procedure was used in a population average model for variable selection. Since a large number of observations were available, a $p$ value of $\leq 0.1$ was used for entry and retention in the model. The variables that were retained in the population average model were thereafter introduced into a model using generalized estimating equations (GEE) to control for multiple isolates from each faecal sample [15]. This was performed with a repeated measure on calf at each sampling occasion with an independent correlation matrix design. The proportional odds assumption was assessed by the score statistic and by graphing odds ratios for sampling and treatment group at each level in the cluster hierarchy and assessing trends. The final model was described by the following equation: Logit(cluster) $=\beta_{0}+\beta_{1}($ abtx $)+\beta_{2}$ ( source) $+\beta_{3}$ (wean) $+\beta_{4}$ (time) $+\beta_{5}$ $($ abtx $\times$ time $)+\beta_{6}($ source $\times$ time $)+\beta_{7}$ $($ abtx $\times$ source $) /($ repeated sampling within calf $\times$ time)

\section{RESULTS}

\subsection{Overview}

During the study, no animal in the sampling cohort became ill or necessitated antimicrobial treatment. Of the total 370 cattle received, only 1 animal (not sampled in this study) required treatment for illness between arrival and study end.

In total, 3179 isolates were obtained from the steers. Of the 49 isolates biochemically tested, 48 (98\%) produced reactions typical of $E$. coli. The inhibition zones for most antimicrobials demonstrated a bimodal distribution. All isolates were sensitive to nalidixic acid. Since antimicrobial susceptibility tests to nalidixic acid exhibited no bimodal distribution, they were not included as variables to determine antimicrobial resistance cluster membership, but are included in description of clusters.

\subsection{Antimicrobial resistance clusters}

The E. coli antimicrobial resistance cluster characteristics and number of isolates belonging to each cluster are shown in Table II. Clusters were optimized to contain minimal intra-cluster variability to the eleven antimicrobials. The optimal number of clusters was based upon having isolates with disk diffusion zones only in one zone of the bimodal distribution. Thirteen clusters adequately described the major antimicrobial resistance patterns and were ordered in increasing levels of resistance from A to M. Of the 3179 isolates, 34 (about $1 \%$ of the dataset) were trimmed during the clustering algorithm, leaving 3145 isolates for analysis. The majority of isolates $(82 \%)$ belonged to cluster A, containing bacteria susceptible to the 11 antimicrobials. Tetracycline resistance was seen in the remaining clusters (B$\mathrm{M})$ combined with other resistance to one or several antimicrobials. Chloramphenicol resistance, which is present in $E$. coli when florfenicol resistance is present, was seen in cluster D, G and I-M.

\subsection{Statistical analysis}

A contingency table of $E$. coli cluster membership by sampling time and source is shown (Tab. III). The influence of florfenicol treatment on E. coli cluster membership was assessed (Tab. IV). Treated steers displayed a significantly greater proportion of isolates with chloramphenicol resistance than non-treated steers at sampling days 14 , 28 and 42 . On the second sampling, occurring 3 days post-treatment (day 14), all isolates obtained from treated cattle belonged to clusters containing chloramphenicol resistance, compared to only $1 \%$ of isolates from non-treated steers. Though less pronounced in the third and fourth sampling, treated cattle had significantly greater proportion of 
Table III. Percentage of faecal E. coli isolates (total number $=3145$ ) by source group (A and B), sampling occasion (day 1, 14, 28 and 42), and antimicrobial resistance cluster membership in a trial evaluating single-dose florfenicol treatment of steers.

\begin{tabular}{|c|c|c|c|c|c|c|c|c|c|}
\hline \multirow{3}{*}{ Cluster } & \multirow{3}{*}{ Frequency } & \multicolumn{8}{|c|}{ Percentage of E. coli isolates by source, sampling, and cluster membership } \\
\hline & & \multicolumn{2}{|c|}{ Day $1^{*}$} & \multicolumn{2}{|c|}{ Day $14 * *$} & \multicolumn{2}{|c|}{ Day $28 * *$} & \multicolumn{2}{|c|}{ Day $42 * *$} \\
\hline & & A & $\mathrm{B}$ & A & B & A & B & A & B \\
\hline A & 2518 & 97 & 76 & 73 & 64 & 85 & 81 & 88 & 87 \\
\hline B & 263 & 3 & 6 & 2 & 5 & 6 & 13 & 6 & 10 \\
\hline C & 23 & 0 & 3 & 0 & 0 & 2 & 0 & 0 & 0 \\
\hline D & 5 & 0 & 0 & 1 & 0 & 0 & 0 & 0 & 0 \\
\hline E & 54 & 0 & 6 & 0 & 3 & 0 & 2 & 1 & 1 \\
\hline F & 31 & 0 & 5 & 0 & 2 & 0 & 2 & 0 & 0 \\
\hline G & 71 & 0 & 0 & 3 & 7 & 4 & 2 & 2 & 1 \\
\hline $\mathrm{H}$ & 12 & 0 & 3 & 0 & 0 & 0 & 0 & 0 & 1 \\
\hline I & 12 & 0 & 0 & 0 & 3 & 0 & 0 & 0 & 0 \\
\hline $\mathrm{J}$ & 8 & 0 & 0 & 0 & 2 & 2 & 0 & 0 & 0 \\
\hline $\mathrm{K}$ & 31 & 0 & 0 & 17 & 5 & 0 & 0 & 3 & 0 \\
\hline $\mathrm{L}$ & 107 & 0 & 0 & 1 & 7 & 0 & 0 & 0 & 0 \\
\hline $\mathrm{M}$ & 10 & 0 & 0 & 2 & 1 & 0 & 0 & 0 & 0 \\
\hline
\end{tabular}

* Source group B had significantly higher proportion of isolates belonging to more resistant clusters than group A (Wilcoxon-Mann-Whitney test, $p$-value $<0.0001$ ).

** No significant difference in resistance between group A and B at day 14, 28 and 42, Wilcoxon-MannWhitney test $p$-value $=0.16,0.23$ and 0.46 respectively.

isolates belonging to clusters with chloramphenicol resistance. Apart from the $1 \%$ of isolates belonging to chloramphenicol resistant cluster $\mathrm{D}$ found at Day 14 , there was no indication of chloramphenicol resistance in control steers. The faecal $E$. coli isolates obtained from non-treated steers housed in pens with treated steers compared to nontreated steers housed in pens with only nontreated steers revealed no significant difference in cluster distribution (WilcoxonMann-Whitney test $p$-value $=0.46$ )

The multinomial logistic regression model showed that treatment group, sampling time and animal source, as well as interactions between these variables, were important predictors of the odds of $E$. coli belonging to a more resistant cluster (Tab. V). There was no association with nutrition treatment and pen mates to treated steers did not show increasing levels of resistance compared to steers in pens without treatment.

The main effect of treatment indicates a clear shift toward carriage of more resistant E. coli in calves treated with florfenicol. The statistical interactions indicate an effect-measure modification. In order to determine the probability of a steer belonging to a higher order cluster, the log-odds of all variables, main effects and interactions, must be calculated. As a result, the GEE estimate of $\log$ odds ratio associated with single main effect variables cannot be simply interpreted as the magnitude of effect. The model is therefore discussed in terms of trends for different variables. Abtx $\times$ time interaction formally indicated what was 
Table IV. Percentage faecal E. coli isolates (total number $=3145$ ) belonging to antimicrobial resistance clusters A-M from steers in a clinical trial having received a single-dose florfenicol treatment on study day 11 (abtx) group and control group by sampling occasion (day 1, 14, 28 and 42).

Percentage of E. coli isolates by treatment group, sampling time,

and cluster membership

\begin{tabular}{|c|c|c|c|c|c|c|c|c|c|}
\hline \multirow[t]{2}{*}{ Cluster } & \multirow[t]{2}{*}{ Frequency } & \multicolumn{2}{|c|}{ Day 1} & \multicolumn{2}{|c|}{ Day $14 *$} & \multicolumn{2}{|c|}{ Day 28* } & \multicolumn{2}{|c|}{ Day $42 *$} \\
\hline & & control & abtx & control & abtx & control & abtx & control & abtx \\
\hline A & 2518 & 88 & 87 & 91 & 0 & 87 & 72 & 88 & 83 \\
\hline B & 263 & 5 & 5 & 5 & 0 & 10 & 8 & 9 & 5 \\
\hline $\mathrm{C}$ & 23 & 2 & 0 & 0 & 0 & 2 & 0 & 0 & 0 \\
\hline $\mathbf{D}^{* *}$ & 5 & 0 & 0 & 1 & 0 & 0 & 0 & 0 & $\mathbf{0}$ \\
\hline E & 54 & 3 & 3 & 2 & 0 & 1 & 2 & 2 & 1 \\
\hline $\mathrm{F}$ & 31 & 3 & 0 & 1 & 0 & 1 & 0 & 0 & 0 \\
\hline $\mathbf{G}^{* *}$ & 71 & 0 & 0 & 0 & 20 & 0 & 12 & 0 & 6 \\
\hline $\mathrm{H}$ & 12 & 0 & 4 & 0 & 0 & 0 & 0 & 1 & 0 \\
\hline I** & 12 & 0 & $\mathbf{0}$ & 0 & 7 & 0 & 0 & $\mathbf{0}$ & $\mathbf{0}$ \\
\hline $\mathbf{J}^{* *}$ & 8 & $\mathbf{0}$ & $\mathbf{0}$ & $\mathbf{0}$ & 4 & $\mathbf{0}$ & $\mathbf{0}$ & $\mathbf{0}$ & $\mathbf{0}$ \\
\hline $\mathbf{K}^{* *}$ & 31 & 0 & $\mathbf{0}$ & 0 & 17 & 0 & 1 & $\mathbf{0}$ & $\mathbf{0}$ \\
\hline $\mathbf{L}^{* *}$ & 107 & $\mathbf{0}$ & $\mathbf{0}$ & $\mathbf{0}$ & 47 & $\mathbf{0}$ & 6 & $\mathbf{0}$ & 6 \\
\hline $\mathbf{M}^{* *}$ & 10 & 0 & $\mathbf{0}$ & $\mathbf{0}$ & 6 & $\mathbf{0}$ & $\mathbf{0}$ & $\mathbf{0}$ & $\mathbf{0}$ \\
\hline
\end{tabular}

* Steers receiving florfenicol treatment on day 11 had a significantly greater proportion of isolates belonging to clusters with chloramphenicol resistance than non-treated steers (Chi-square $p$-value $<$ $0.05)$.

** Clusters containing chloramphenicol resistance.

evident in the contingency tables, that $E$. coli isolates of treated calves tended to return to less resistant cluster groupings as time from treatment increased.

Calves from source B tended to carry a greater proportion of resistant $E$. coli than calves from source A. Furthermore, calves from source B that were weaned one month prior to shipment carried a higher proportion of resistant $E$. coli compared to calves gathered up directly from pasture.

Over time, E. coli antimicrobial resistance between sources moved toward a common profile. Though source A steers displayed a greater proportion of susceptible $E$. coli initially, time $\times$ source interaction indicated that source A tended to display more resistance over time, relative to source $B$ steers. Furthermore, abtx $\times$ source interac- tion indicates source A calves tended to carry a greater proportion of resistant $E$. coli due to treatment than calves from source B.

\section{DISCUSSION}

Florfenicol is a fluorinated analogue of chloramphenicol that was approved in 1996 in the USA for bovine respiratory disease pathogens. It is routinely used in the USA feedlot industry and has been shown to be clinically successful in treating respiratory disease and fever [4]. It has broad spectrum activity, including respiratory pathogens and gram-negative bacterial flora, and has an extended duration of action. It is found in high concentration in urine and bile, and would be expected to impact faecal $E$. coli flora [2]. Chloramphenicol, the parent 
Table V. Cumulative logistic model: GEE Estimates of log odds faecal E. coli isolates belonging to clusters with higher level antimicrobial resistance in a trial evaluating single-dose florfenicol treatment of steers on antimicrobial resistance in faecal E. coli.

\begin{tabular}{|c|c|c|c|c|c|c|c|c|}
\hline & & $\begin{array}{c}\text { GEE } \\
\text { estimate }\end{array}$ & $\begin{array}{c}\text { Standard } \\
\text { error }\end{array}$ & Df & $\begin{array}{l}\text { Lower } \\
\text { C.I. }\end{array}$ & $\begin{array}{l}\text { Upper } \\
\text { C.I. }\end{array}$ & $\begin{array}{l}\text { Chi- } \\
\text { square }\end{array}$ & $P$-value \\
\hline \multicolumn{9}{|c|}{ Treatment (abtx) } \\
\hline \multicolumn{2}{|c|}{ No } & Reference & & & & & & \\
\hline \multicolumn{2}{|l|}{ Yes } & 6.34 & 0.66 & 1 & 5.05 & 7.63 & 9.64 & $<0.0001$ \\
\hline \multicolumn{9}{|c|}{ Sampling time (time) } \\
\hline \multicolumn{2}{|c|}{1} & Reference & & & & & & \\
\hline \multicolumn{2}{|l|}{2} & -0.80 & 0.39 & 1 & -1.57 & -0.03 & -2.03 & 0.0427 \\
\hline \multicolumn{2}{|l|}{3} & -0.52 & 0.40 & 1 & -1.31 & 0.27 & -1.29 & 0.1962 \\
\hline \multicolumn{2}{|l|}{4} & -0.76 & 0.39 & 1 & -1.53 & 0.01 & -1.93 & 0.0542 \\
\hline \multicolumn{9}{|c|}{ Source of steers (source) } \\
\hline \multicolumn{2}{|c|}{$\mathrm{B}$} & Reference & & & & & & \\
\hline \multicolumn{2}{|l|}{ A } & -2.07 & 0.41 & 1 & -2.87 & -1.28 & -5.1 & $<0.0001$ \\
\hline \multicolumn{9}{|c|}{ Weaned one month prior shipment (wean) } \\
\hline \multicolumn{2}{|c|}{ Yes } & Reference & & & & & & \\
\hline \multicolumn{2}{|l|}{ No } & -0.59 & 0.29 & 1 & -1.16 & -0.02 & -2.04 & 0.0416 \\
\hline \multicolumn{9}{|c|}{ Interaction time and source } \\
\hline \multicolumn{2}{|c|}{ Time $=4 \quad$ Source A } & 1.88 & 0.53 & 1 & 0.83 & 2.92 & 3.52 & 0.0004 \\
\hline \multirow{3}{*}{$\begin{array}{l}\text { Time }=4 \\
\text { Time }=3 \\
\text { Time }=3\end{array}$} & Source B & Reference & & & & & & \\
\hline & Source A & 1.69 & 0.57 & 1 & 0.57 & 2.80 & 2.96 & 0.003 \\
\hline & Source B & Reference & & & & & & \\
\hline Time $=2$ & Source A & 1.30 & 0.62 & 1 & 0.09 & 2.51 & 2.1 & 0.0357 \\
\hline Time $=2$ & Source B & Reference & & & & & & \\
\hline \multirow{2}{*}{$\begin{aligned} \text { Time } & =1 \\
\text { Time } & =1\end{aligned}$} & Source A & Reference & & & & & & \\
\hline & Source B & Reference & & & & & & \\
\hline \multicolumn{9}{|c|}{ Interaction abtx and source } \\
\hline$a b t x=1$ & Source A & 1.31 & 0.48 & 1 & 0.36 & 2.25 & 2.72 & 0.0065 \\
\hline abtx $=1$ & Source B & Reference & & & & & & \\
\hline \multirow{2}{*}{$\begin{array}{l}\text { abtx }=0 \\
\text { abtx }=0\end{array}$} & Source A & Reference & & & & & & \\
\hline & Source B & Reference & & & & & & \\
\hline \multicolumn{9}{|c|}{ Interaction abtx and time } \\
\hline abtx $=$ yes & Time $=4$ & -6.51 & 0.85 & 1 & -8.17 & -4.84 & -7.66 & $<0.0001$ \\
\hline abtx $=$ yes & Time $=3$ & -5.89 & 0.75 & 1 & -7.36 & -4.43 & -7.9 & $<0.0001$ \\
\hline abtx $=$ yes & Time $=2$ & Reference & & & & & & \\
\hline $\mathrm{abtx}=$ no & Time $=4$ & Reference & & & & & & \\
\hline abtx $=$ no & Time $=3$ & Reference & & & & & & \\
\hline abtx $=$ no & Time $=2$ & Reference & & & & & & \\
\hline abtx $=$ no & Time $=1$ & Reference & & & & & & \\
\hline
\end{tabular}


compound of florfenicol, was prohibited from use in food animals in 1986.

This study has demonstrated a very clear but transitory shift in antimicrobial resistance patterns of faecal $E$. coli of feedlot steers in response to systemic florfenicol treatment. Results here indicate that florfenicol selects for exclusively resistant faecal flora three days post treatment, during the period when the antimicrobial would be expected to be present in the feces. This suggests that post-therapy, treated animals shed a larger proportion of resistant organisms in their faeces.

Treated steers had a significantly higher proportion of isolates belonging to clusters with chloramphenicol resistance, compared to non-treated steers. We hypothesize that this is due to selection of $E$. coli with a plasmid or chromosomally located flo gene conferring resistance to florfenicol and chloramphenicol [27,33]. Additionally, florfenicol treatment selected for $E$. coli isolates that exhibited simultaneous resistance to several other antimicrobial classes. We hypothesize that this resistance co-selection is due to linkage of antimicrobial resistance genes. E. coli have been shown to harbour a large array of plasmids with different antimicrobial resistance genes. Florfenicol treatment likely selected for $E$. coli with plasmids containing multiple antimicrobial resistance genes. Several plasmid types carrying the flo gene in $E$. coli isolates of bovine origin have been identified, and multiple resistance was a common feature [7].

Whether multi-resistant $E$. coli persist in the environment, or establish in non-treated animals following casual contact is not known. The 21 untreated pen mates of treated cattle showed no tendency to shed faecal E. coli with higher levels of resistance. This finding suggested that the environmental spread of resistant bacteria from treated to untreated steers was limited in this study.

Due to limited resources, we did not confirm all isolates as E. coli, so a small proportion of non-E. coli organisms almost cer- tainly were included in the analysis. Assuming they occurred sporadically and at random, they would not be expected to affect the analysis. Berge et al. demonstrated no difference in antimicrobial resistance pattern distribution in non- $E$. coli isolated from MacConkey agar compared to isolates biochemically confirmed as E. coli [5].

Our study has shown that the antimicrobial resistance associated with florfenicol treatment decreased dramatically over 4 weeks post-treatment. Similar to other range beef populations, this population of steers had generally susceptible flora at sample day 1. It appears that antimicrobial treatment as described here did not have a large long-term effect on $E$. coli resistance pattern of treated cattle. By study end, some resistant organisms were evident in treated steers, compared to controls. The significance of this small proportion, about $6 \%$ of E. coli, is unknown. It is unknown what effect another antimicrobial treatment, florfenicol or other, would have in treated steers.

Use of cluster analysis allowed descriptive assessment of antimicrobial susceptibility to multiple antimicrobials. As a result of florfenicol treatment, resistance to chloramphenicol was observed, as well as resistance to other antimicrobials. Higher order clusters were associated with higher orders of resistance, and florfenicol treatment was associated with selection of $E$. coli possessing chloramphenicol, tetracycline, sulfisoxazole, streptomycin, and some beta-lactam resistance. It is unknown if the resistance observed was transferable, so it is not possible to fully assess the direct implication of this observation.

As indicated by the stratified analysis and multinomial model, antibiotic treatment had a strong effect on E. coli antimicrobial resistance patterns. However, the model and stratified analysis both indicated that $E$. coli antimicrobial resistance patterns in treated steers moved back toward baseline levels over time. Resistant bacteria may incur a fitness cost to retain resistance genes 
in the absence of further antibiotic selective pressure [21]. At the end of our study a higher proportion of faecal E. coli were resistant than at the time of arrival. It is unknown whether this proportion would further decrease over time, or stabilize at this level.

Though of lesser magnitude, environmental factors influenced level of antimicrobial resistance following treatment. Source differences are intriguing, in that they suggest some attribute or dynamic that existed at arrival and impacted subsequent antimicrobial resistance cluster membership. Though individual antimicrobial use records were not available from ranch sources, antimicrobials were not included in feed prior to feedlot entry. Additionally, ranch personnel indicated that use of antimicrobials for individual animal treatment was very infrequent. The difference between source A and source B was not solely attributed to the fact that some calves were weaned and rounded up one month prior shipment, but that other environmental factors were also involved. It is unknown how these effects would appear after a longer time in the feedlot.

Though E. coli of source A steers appeared more susceptible initially, they paradoxically tended to shift to higher levels of resistance with time, compared to source B. In a similar manner, swine not exposed to antimicrobials as piglets, then exposed subsequently showed a similar response, compared to swine exposed to antimicrobials early in life [10]. Initial antimicrobial susceptibility differences observed between sources decreased over time in the feedlot, as proportions of isolates in source $\mathrm{A}$ and source B calves appeared to become similar. Changes observed over time between sources reflect a natural selection toward a uniform flora. This flora is apparently influenced by factors apart from antimicrobial use, possibly including feedstuffs, feed management, and pen environment $[9,16$, 20]. This observation has ramifications in future studies, and suggests that source may impact faecal $E$. coli resistance patterns, but that these patterns will tend to migrate toward the patterns dictated by feedlot environment. This suggests that antimicrobial resistance of feedlot cattle may be partially manipulated by management interventions apart from controlling antimicrobial use in the feedlot, and may suggest that antimicrobial resistance of enteric bacteria in animals not exposed to antimicrobials may still exhibit changes over time [22].

\section{ACKNOWLEDGEMENTS}

Supported by the Ethel Austin Martin Program for Human Nutrition (South Dakota State University, Brookings, SD, 57007, USA). Special thanks to C.A. Gates for technical assistance.

\section{REFERENCES}

[1] Anonymous, Performance standards for antimicrobial disk and dilution susceptibility tests for bacteria isolated from animals; Approved standard, 2nd ed., National Committee for Clinical Laboratory Standards, Wayne, Pennsylvania, 2002.

[2] Adams P.E., Varma K.J., Powers T.E., Lamendola J.F., Tissue concentrations and pharmacokinetics of florfenicol in male veal calves given repeated doses, Am. J. Vet. Res. 48 (1987) 1725-1732.

[3] Agresti A., Categorical data analysis, John Wiley \& Sons, Inc., New York, 2002.

[4] Bauer A.W., Kirby W.M., Sherris J.C., Turck M., Antibiotic susceptibility testing by a standardized single disk method, Am. J. Clin. Pathol. 45 (1966) 493-496.

[5] Berge A.C.B., Atwill E.R., Sischo W.M., Assessing antibiotic resistance in fecal Escherichia coli in young calves using cluster analysis techniques, Prev. Vet. Med. 61 (2003) 91-102.

[6] Blake D.P., Hillman K., Fenlon D.R., Low J.C., Transfer of antibiotic resistance between commensal and pathogenic members of the Enterobacteriaceae under ileal conditions, J. Appl. Microbiol. 95 (2003) 428-436. 
7] Cloeckaert A., Baucheron S., Chaslus-Dancla E., Nonenzymatic chloramphenicol resistance mediated by IncC plasmid R55 is encoded by a floR gene variant, Antimicrob. Agents Chemother. 45 (2001) 2381-2382.

[8] Courvalin P., Transfer of antibiotic resistance genes between gram-positive and gram-negative bacteria, Antimicrob. Agents Chemother. 38 (1994) 1447-1451.

[9] Dargatz D.A., Fedorka-Cray P.J., Ladely S.R., Ferris K.E., Green A.L., Headrick M.L., Antimicrobial susceptibility patterns of Salmonella isolates from cattle in feedlots, J. Am. Vet. Med. Assoc. 221 (2002) 268-272.

[10] Dawson K.A., Langlois B.E., Stahly T.S., Cromwell G.L., Antibiotic resistance in anaerobic and coliform bacteria from the intestinal tract of swine fed therapeutic and subtherapeutic concentrations of chlortetracycline, J. Anim. Sci. 58 (1984) 123-131.

[11] DeFrancesco K.A., Cobbold R.N., Rice D.H., Besser T.E., Hancock D.D., Antimicrobial resistance of commensal Escherichia coli from dairy cattle associated with recent multiresistant salmonellosis outbreaks, Vet. Microbiol. 98 (2004) 55-61.

[12] Everitt B.S., Cluster analysis, Halsted Press, John Wiley \& Sons Inc., New York, 1993.

[13] Food and Drug Administration, Nuflor ${ }^{\circledR}$ (florfenicol), FOI summary, NADA 141-063 (original), Laurel, Md, 1996.

[14] Hagedorn C., Robinson S.L., Filtz J.R., Grubbs S.M., Angier T.A., Reneau R.J., Determining sources of fecal pollution in a rural Virginia watershed with antibiotic resistance patterns in fecal streptococci, Appl. Environ. Microbiol. 65 (1999) 5522-5531.

[15] Hanley J.A., Statistical analysis of Correlated Data using GEE, An orientation, Am. J. Epidemiol. 157 (2003) 364-375.

[16] Hinton M., Rixson P.D., Allen V., Linton A.H., The persistence of drug resistant Escherichia coli strains in the majority faecal flora of calves, J. Hyg. (Lond.) 93 (1984) 547557.

[17] Hoyle D.V., Knight H.I., Shaw D.J., Hillman K., Pearce M.C., Low J.C., Gunn G.J., Woolhouse M.E., Acquisition and epidemiology of antibiotic-resistant Escherichia coli in a cohort of newborn calves, J. Antimicrob. Chemother. 53 (2004) 867-871.

[18] Khachatryan A.R., Hancock D.D., Besser T.E., Call D.R., Role of calf-adapted Escherichia coli in maintenance of antimicro- bial drug resistance in dairy calves, Appl. Environ. Microbiol. 70 (2004) 752-757.

[19] Kleinbaum D.G., Kupper L.L., Muller K.E., Nizam A., Applied regression analysis and other multivariable methods, Duxbury Press, Pacific Grove, CA, 1998, pp. 1-718.

[20] Langlois B.E., Dawson K.A., Leak I., Aaron D.K., Antimicrobial resistance of fecal coliforms from pigs in a herd not exposed to antimicrobial agents for 126 months, Vet. Microbiol. 18 (1988) 147-153.

[21] Lenski R.E., The cost of antibiotic resistance - from the perspective of a bacterium, Ciba Found. Symp. 207 (1997) 131-140.

[22] Marshall B., Petrowski D., Levy S.B., Interand intraspecies spread of Escherichia coli in a farm environment in the absence of antibiotic usage, Proc. Natl. Acad. Sci. USA 87 (1990) 6609-6613.

[23] Mathew A.G., Saxton A.M., Upchurch W.G., Chattin S.E., Multiple antibiotic resistance patterns of Escherichia coli isolates from swine farms, Appl. Environ. Microbiol. 65 (1999) 2770-2772.

[24] McEwen S.A., Fedorka-Cray P.J., Antimicrobial use and resistance in animals, Clin. Infect. Dis. 34 Suppl. 3 (2002) S93-S106.

[25] Phillips I., Casewell M., Cox T., De Groot B., Friis C., Jones R., Nightingale C., Preston R., Waddell J., Does the use of antibiotics in food animals pose a risk to human health? A critical review of published data, J. Antimicrob. Chemother. 53 (2004) 28-52.

[26] Saenz Y., Zarazaga M., Lantero M., Gastanares M.J., Baquero F., Torres C., Antibiotic resistance in Campylobacter strains isolated from animals, foods, and humans in Spain in 1997-1998, Antimicrob. Agents Chemother. 44 (2000) 267-271.

[27] Singer R.S., Patterson S.K., Meier A.E., Gibson J.K., Lee H.L., Maddox C.W., Relationship between phenotypic and genotypic florfenicol resistance in Escherichia coli, Antimicrob. Agents Chemother. 48 (2004) 4047-4049.

[28] Smith D.L., Harris A.D., Johnson J.A., Silbergeld E.K., Morris J.J., Animal antibiotic use has an early but important impact on the emergence of antibiotic resistance in human commensal bacteria, Proc. Natl. Acad. Sci. USA 99 (2002) 6434-6439.

[29] Stabler S.L., Fagerberg D.J., Quarles C.L., Effects of oral and injectable tetracyclines on 
bacterial drug resistance in feedlot cattle, Am. J. Vet. Res. 43 (1982) 1763-1766.

[30] van den Bogaard A.E. Stobberingh E.E., Epidemiology of resistance to antibiotics. Links between animals and humans, Int. J. Antimicrob. Agents 14 (2000) 327-335.

31] Wagner B.A., Dargatz D.A., Salman M.D., Morley P.S., Wittum T.E., Keefe T.J., Comparison of sampling techniques for measuring the antimicrobial susceptibility of enteric
Escherichia coli recovered from feedlot cattle, Am. J. Vet. Res. 63 (2002) 1662-1670.

[32] Ward J.H., Hierarchical grouping to optimize an objective function, J. Am. Stat. Assoc. 58 (1963) 236-244.

[33] White D.G., Hudson C., Maurer J.J., Ayers S., Zhao S., Lee M.D., Bolton L., Foley T., Sherwood J., Characterization of chloramphenicol and florfenicol resistance in Escherichia coli associated with bovine diarrhea, J. Clin. Microbiol. 38 (2000) 4593-4598. 